\title{
MULTICHANNEL ANALYSIS OF EEG SIGNALS FOR SCREENING ALCOHOLICS
}

\author{
By \\ Jasmin Thevaril
}

\begin{abstract}
A Project
SUBMITTED IN PARTIAL FULFILLMENT OF THE

REQUIREMENTS FOR THE DEGREE OF

MASTER OF ENGINEERING

AT

RYERSON UNIVERSITY

350 VICTORIA STREET, TORONTO, ONTARIO, CANADA

MAY 2004
\end{abstract}

Copyright by Jasmin Thevaril, 2004 
UMI Number: EC52981

\section{All rights reserved \\ INFORMATION TO USERS}

The quality of this reproduction is dependent upon the quality of the copy submitted. Broken or indistinct print, colored or poor quality illustrations and photographs, print bleed-through, substandard margins, and improper alignment can adversely affect reproduction.

In the unlikely event that the author did not send a complete manuscript and there are missing pages, these will be noted. Also, if unauthorized copyright material had to be removed, a note will indicate the deletion.

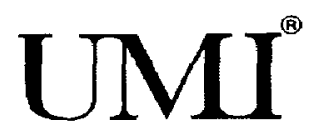

UMI Microform EC52981

Copyright 2008 by ProQuest LLC

All rights reserved. This microform edition is protected against unauthorized copying under Title 17, United States Code.

ProQuest LLC

789 East Eisenhower Parkway

P.O. Box 1346

Ann Arbor, Ml 48106-1346 


\section{RYERSON UNIVERSITY \\ DEPARTMENT OF \\ ELECTRICAL AND COMPUTER ENGINEERING}

The undersigned hereby certify that they have read and recommend to the Faculty of Graduate Studies for acceptance a thesis entitled "Multichannel Analysis of EEG Signals for Screening Alcoholics" by Jasmin Thevaril in partial fulfillment of the requirements for the degree of Master of Engineering.

Dated: May 2004

Supervisor:

Dr. S. Krishnan

Readers: 


\section{RYERSON UNIVERSITY}

Date: May 2004

Author: Jasmin Thevaril

Title: Multichannel Analysis of EEG Signals for Screening Alcoholics

Department: Electrical and Computer Engineering

Degree: MEng Convocation: June Year: 2004

Permission is herewith granted to Ryerson University to circulate and to have copied for non-commercial purposes, at its discretion, the above title upon the request of individuals or institutions.

Signature of Author

THE AUTHOR RESERVES OTHER PUBLICATION RIGHTS, AND NEITHER THE THESIS NOR EXTENSIVE EXTRACTS FROM IT MAY BE PRINTED OR OTHERWISE REPRODUCED WITHOUT THE AUTHOR'S WRITTEN PERMISSION.

THE AUTHOR ATTESTS THAT PERMISSION HAS BEEN OBTAINED FOR THE USE OF ANY COPYRIGHTED MATERIAL APPEARING IN THIS THESIS (OTHER THAN BRIEF EXCERPTS REQUIRING ONLY PROPER ACKNOWLEDGEMENT IN SCHOLARLY WRITING) AND THAT ALL SUCH USE IS CLEARLY ACKNOWLEDGED. 


\section{Table of Contents}

Table of Contents

Abstract $\quad$ viii

$\begin{array}{ll}\text { Acknowledgements } & \text { ix }\end{array}$

1 INTRODUCTION 1

1.1 INTRODUCTION TO ALCOHOLISM . . . . . . . . . . . . . 1

1.2 AN OVERVIEW OF EEG . . . . . . . . . . . . . . . 2

1.2 .1 EEG FEATURES . . . . . . . . . . . . . . . 3

1.2 .2 EEG DATA ACQUISITION . . . . . . . . . . . . 4

1.3 EEG SIGNAL ANALYSIS . . . . . . . . . . . . . . 5

1.4 ADVANTAGES OF USING EEG . . . . . . . . . . . . 6

1.5 APPLICATIONS OF EEG . . . . . . . . . . . . . . 6

1.5.1 DIAGNOSIS OF BRAIN DISORDERS . . . . . . . . . 6

1.6 MOTIVATION ........................ . . . 7

1.6 .1 OBJECTIVE .................... 8

1.7 PREVIEW OF THE REPORT $\ldots \ldots \ldots \ldots$

2 REVIEW

2.1 NON-INVASIVE DIAGNOSIS USING EEG . . . . . . . . . . . 11

2.1 .1 EEG SIGNAL ANALYSIS . . . . . . . . . . . . . . 11

2.1.2 SCREENING TECHNIQUES FOR ANY DISORDER . . . . 13

2.1 .3 HJORTH ANALYSIS . . . . . . . . . . . . . . . . . . 13

2.1 .4 AUTO-REGRESSIVE ANALYSIS . . . . . . . . . . . 14

2.1.5 SPECTRAL PARAMETER ANALYSIS(SPA) . . . . . . . 15

2.2 EEG IN ALCOHOLISM . . . . . . . . . . . . . . . . 16

2.3 MULTI-CHANNEL ANALYSIS OF EEG . . . . . . . . . . . . . 17

2.3.1 MULTICOMPONENT CHARACTERISTICS OF EEG . . . . 21 
3 METHODOLOGY 23

3.1 SEGMENTATION OF EEG . . . . . . . . . . . . . . . . . 23

3.2 SPECTRAL ESTIMATION OF THE SIGNAL . . . . . . . . . 25

3.3 DESCRIPTION OF AR MODEL . . . . . . . . . . . . . . 28

3.4 CEPTSTRAL THEORY . . . . . . . . . . . . . . . . 31

4 PATTERN CLASSIFICATION 35

4.1 LINEAR DISCRIMINANT ANALYSIS (LDA) . . . . . . . . . . 35

4.2 LEAVE ONE OUT ALGORITHM (LWO) . . . . . . . . . . . . 36

4.2 .1 PATTERN ANALYSIS OF EEG SIGNAL . . . . . . . . 38

5 RESULTS AND DISCUSSION $\quad \mathbf{4 0}$

5.1 RESULTS USING FEATURE ANALYSIS . . . . . . . . . . . . . 40

5.2 RESULTS OF PATTERN CLASSIFICATION OF ALCOHOLIC EEG 42

5.3 DISCUSSIONS AND FUTURE WORK . . . . . . . . . . 45

$\begin{array}{ll}\text { A } & \mathbf{4 7}\end{array}$

$\begin{array}{ll}\text { Bibliography } & 48\end{array}$ 


\section{List of Tables}

2.1 Electrode Placement . . . . . . . . . . . . . . . . 18 


\section{List of Figures}

1.1 Frequency ranges . . . . . . . . . . . . . . . . . 3

1.2 Flowchart of the proposed alcoholic screening system . . . . . . 10

2.1 EEG Acquisition $\ldots \ldots \ldots \ldots \ldots \ldots \ldots$

2.2 Different channels of an EEG signal . . . . . . . . . . . . . . 20

2.3 Multichannel EEG signals . . . . . . . . . . . . . . . . . . 22

3.1 Segmentation Chart . . . . . . . . . . . . . . . . . . . . 24

3.2 Transformation of alcoholic EEG segment using FFT . . . . . . . 26

3.3 Periodogram . . . . . . . . . . . . . . . . 26

3.4 Pole-zero plot . . . . . . . . . . . . . . . . 27

3.5 Cepstral Analysis . . . . . . . . . . . . . . . . . . . 31

3.6 Real Ceptrum . . . . . . . . . . . . . . . . . 33

$5.1 \quad$ Periodogram . . . . . . . . . . . . . . . . . . 41

5.2 EEG channel $17 \ldots \ldots \ldots \ldots$. . . . . . . . . . . . 41

5.3 Classification Summary Results . . . . . . . . . . . . . 42

5.4 Classification Table with AR modeling . . . . . . . . . . . . . 43

5.5 Classification Table with Cepstral Theory . . . . . . . . . . . 44 


\section{Abstract}

Mutli-channel analysis of EEG signals were analyzed in the project to detect alcoholism. A digital signal-processing algorithm that automates the classification of signals as normal or alcoholic is studied here. The method exploits the existing digital signal processing concepts such as signal modeling and spectral estimation for feature extraction and classification. The multi-channel AR modeling and Cepstral theory were used for feature extraction. The EEG signals used in the project include 32 channels recorded from different portions of the brain for three minutes duration. 25 signals of each subject were taken for analysis. A classifier is developed based on Linear Discriminant Analysis (LDA) and Leave-One-Out method (LWO). The feature extracted from all the signals are included in the database and have to reference vectors as "normal" or "abnormal" signal. First, the feature vector was computed using the LDA method. Then by using Leave-One-Out Algorithm (LWO), the signal features were classified based on the norm distances to maximize the accuracy. Maximum Likelihood Method (MLM) or Euclidean distance is used to extract the norm distance between the signal under test and the reference vector. This was repeated for the entire training database. The classifier thus obtained gave the overall accuracy rate of the system. The accuracy rate obtained with AR coefficients is $72 \%$ and the accuracy rate with cepstral coefficients is $62 \%$ 


\section{Acknowledgements}

I would like to thank Dr. S. Krishnan, my supervisor, for his constant willingness to provide advice and guidance towards the success of this project. Without his continuous help this work would never have come into existence.

I am grateful to my parents for their constant support and encouragement. I am also thankful to all my friends and associates who directly and indirectly helped me in completing this project.

Toronto, Ontario

Jasmin Thevaril

May, 2004 


\section{Chapter 1}

\section{INTRODUCTION}

The purpose of this chapter is to provide an introduction and overview of this project. The chapter provides an introduction to alcoholism, some background information about the EEG signals, motivation and objective of this project. Finally the chapter concludes with a preview of the report.

\subsection{INTRODUCTION TO ALCOHOLISM}

Alcoholism is a chronic, often progressive brain disease with symptoms that include strong need to consume alcohol despite negative consequences, such as serious health problems. Like many other diseases, it has a generally predictable course, has recognized symptoms, and is influenced by both genetic and environmental factors. It leads to many premature deaths, disrupts the life of many people and also elevates yearly medical bills.

All brain functions involve communication among nerve cells in the brain. This communication involves both 'sending' and 'receiving' neurons that communicate very frequently across them called synapses. Messages are carried across synapses 
by chemicals called neurotransmitters. Alcohol physically changes it by affecting communication between neurons like intoxication and sleepiness. After its release, a neurotransmitter crosses the synapse and activates a receptor protein in the receiving neuron. Activating a receptor can cause the receiving neuron to change. Prolonged or repeated exposure to alcohol can cause such changes in neurons that can lead to the development of alcoholism. Hence, alcohol is a central nervous system depressant. It depresses the areas of the brain that are normally inhibitory. Acute consumption of alcohol causes occurrence of seizures in individuals.

\subsection{AN OVERVIEW OF EEG}

Electroencephalograph (EEG) is a recording of the electric signals produced by the brain. The presence of electrical current in the brain was discovered by an English physician, Richard Caton in 1875 [1]. It was not until 1924 that Hans Berger, a German psychiatrist, used his ordinary radio equipment to amplify the brain's electrical activity so that he could record it on graph paper [2]. Berger noticed that rhythmic changes varied with the individual's state of consciousness.

The pattern of activity changes with the level of a person's arousal - if a person is relaxed, then the EEG has many slow waves; if a person is excited, then the EEG has many fast waves. EEG record is presumed to represent only cerebral activity. In reality, it includes other electrical activity that is not of cerebral origin. The activity of the brain is the signal of primary concern, and any other activity that appears in the record will deteriorate the brain signal. These undesired activities are called noise or artifacts. Artifact may mimic abnormalities so it is imperative that sources 


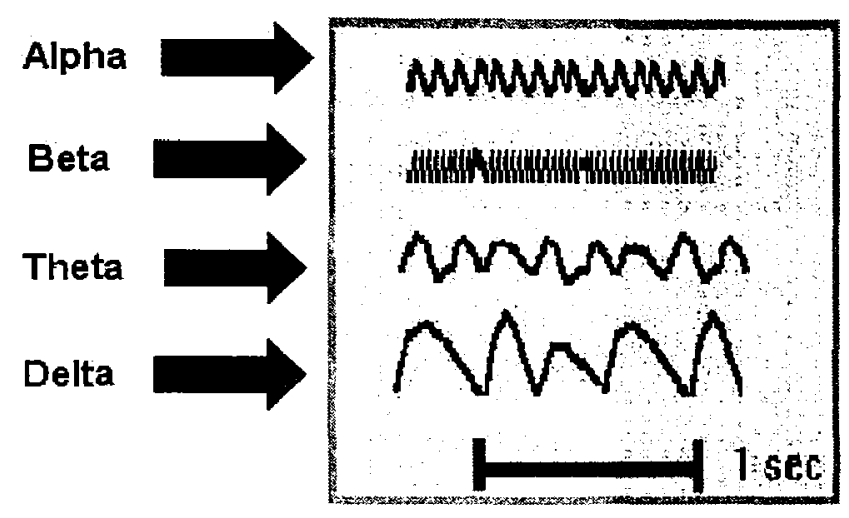

Figure 1.1: Frequency ranges

of noise be identified accurately. An artifact and its cause usually are obvious to the technologist at the time of the recording. But when the EEG is being interpreted, the record may take on an entirely different appearance, in a different setting. Therefore it is essential that artifacts be clearly identified by the technologist and labeled during the time of recording.

\subsubsection{EEG FEATURES}

The variability in EEG signal occurs in two dimensions namely time and amplitude. Amplitude is an index of voltage. The larger the amplitude, the higher the voltage. The voltages in EEG are extremely small. The variability in time is expressed as frequency as shown in Figure 1.1.

EEG reading involves the interpretation of waveforms largely by their frequency and to a lesser extent by the morphology of the wave or of the wave complex of several waves. EEG characteristics are defined by several criteria, some of which are (a) health of the person, (b) age of the person, (c) frequency of observed EEG activity, 
(d) voltage of the observed EEG activity, (e) amount of activity, (f) reactivity of the EEG to various stimuli. Based on these criteria, normal and abnormal activity can be described.

The quantification of EEGs included the classification into four general frequencies: Alpha, Beta, Theta, and Delta as shown in Figure 1.1 below. Alpha waves have relatively large amplitude and moderate frequencies. Beta waves are the result of heightened mental activity; typically show rapid oscillations with small amplitudes. Theta waves accompany feelings of emotional stress, are characterized by moderately low frequencies. Delta waves result from an extremely low frequency oscillation that occurs during periods of deep sleep.

\subsubsection{EEG DATA ACQUISITION}

During the EEG test, a number of electrodes (small silver discs) are attached to the head at specific points using a conductive gel. The doctors may use various procedures (hyperventilation, sleep) during the test to stimulate the brain and trigger certain brain waves.

To obtain the EEG, the activity being produced under one electrode is compared with that of another and plotted out the difference between the two. As brain activity is constantly changing, the signal is recorded over a period of time. In clinical practice, several channels of the EEG are recorded simultaneously from various locations on the scalp. The signals thus recorded are in the analogue format. Then it has to be converted to digital format for applying the various signal processing routines developed using Computer software. 
Data set: All signals used in this project were acquired using the international electrode placement system with a sampling Frequency of $256 \mathrm{~Hz}$. The data set consists of 50 signals with 25 alcoholic and 25 non-alcoholic signals. All signals are recorded for three minutes duration and include 32 channels recorded from different portions of the brain. So there are about 45000 samples. Each file size is approximately 5MB. Eye movement (closed/open) recordings are also included in the evaluation signal set. Usually, there could be considerable redundancy in the EEG, and it is not necessary to review every minute of EEG.

\subsection{EEG SIGNAL ANALYSIS}

The EEG signals are inherently complicated due to their non-Gaussian, non-stationary, and non-linear in nature [2]. On top of that the small amplitude of these signals reinforce their sensitivity to various artifacts and noise sources.

EEG signal usually consists of multiple simultaneous recordings with information from several regions of the brain. Previously, both single and multiple channel type of analysis were considered using signal processing techniques to extract desired information from EEG. There are instances when only a single channel of recording is available. However, generally it is stated that multiple simultaneous recordings of the data are required for information to be extracted through signal processing. Moreover, the signal of interest could be present in a number of channels of a multi-channel recording. This would help to increase the efficiency of detecting the abnormality. On the other side, the difficulty of isolating all channels of interest is then increased. 


\subsection{ADVANTAGES OF USING EEG}

EEG depicts the electrical signals generated on the surface of the brain without opening the skull. It is a completely non-invasive procedure that can be applied repeatedly in patients, normal adults, and children with no risks or limitations. The EEG test is painless. It does not deliver electricity to patient's scalp; it only measures electrical current that is detectable through the skin of patient's scalp. An EEG recorded during a seizure is the best possible way to tell if a seizure is caused by abnormal brain activity. However, the EEG often is normal between seizures making it difficult to reach a conclusive diagnosis.

\subsection{APPLICATIONS OF EEG}

The EEG is widely used in the biomedical field for the detection and treatment of brain disorders, such as epilepsy. It is also used to diagnose the presence and type of seizure disorders, confusion, head injuries, brain tumors, and infections that affect the brain [3]. It is also used to evaluate sleep disorders and to investigate periods of unconsciousness. The EEG test may be done to confirm brain death in a patient.

\subsubsection{DIAGNOSIS OF BRAIN DISORDERS}

EEG activity changes with functional status of the brain such as in sleep, epilepsy [2]. First, the doctor examines the complete EEG record visually. This analysis is performed systematically. The next phase is to decide whether the data is normal or abnormal. If it is abnormal, the type of abnormality should be determined. In this 
process, computers assist the doctor in making the diagnosis of brain disorders easier $[2]$.

There are several ways to diagnose brain disorders using EEG. EEG Neurofeedback is one of them. It is a non-invasive technique that occurs in real time. The information collected is presented back to the person so that they can see the changes in the electrical state of the brain. Using this computerized feedback technology, the brain will get the ability to learn and modify the electrical states, overcome deficiencies. This was originally meant for diseases like stroke and epilepsy. Now this is also used for learning difficulties. Once the brain is trained in a particular state, it is then embedded permanently, just like learning to ride a bike.

\subsection{MOTIVATION}

As indicated, several brain disorders can be detected using EEG signals in a noninvasive manner. Computer-aided diagnosis of the signal helps the clinician in detecting abnormalities in a faster and less tedious manner. By using automated detection the length of hospital stay can be significantly reduced. This project specifically focuses on alcoholism.

Alcohol cravings and several uncontrolled alcohol ingestion are strongly associated with imbalances in certain brain neuro-chemicals. This imbalance is reflected in certain frequency ranges of the EEG in various regions of the brain. The EEG activity is described in terms of several variables with single and multi-channel recordings. To extract maximum information of interest from all regions of the brain affected by the imbalance, a multi-channel signal processing technique is chosen in the project. Again, different parts of the brain do not emit the same brain wave frequency simultaneously. 
So the multiple channels extracted from different parts of the brain are analyzed concurrently for a period of time.

Compression and automatic detection/classification of the signal are the different steps associated with the project, and is described in detail later in the report.

\subsubsection{OBJECTIVE}

The objective of this project is to develop a multi-channel EEG signal analysis technique to automate the process of detecting alcoholism. The EEG signals are to be compressed based on feature extraction and classified using statistical pattern analysis techniques such as Linear Discriminant Analysis using Leave One Out Classification.

\subsection{PREVIEW OF THE REPORT}

This thesis is organized into 5 chapters.

- Chapter 1 is an introductory chapter that gives the reader a brief background information about alcoholism, overview of EEG signals and the overall picture of this project. This chapter also discusses the motivation of the project. The block diagram of the proposed alcoholic screening system is shown in Figure 1.2 .

- Chapter 2 will present a review of related research work done in this area including the scope of multichannel EEG analysis.

- Chapter 3 will explain the theory of the project involved in the signal analysis including spectral estimation, modeling and parametric extraction. 
- The application of the pattern classification techniques to detect multichannel Alcoholic signals is demonstrated in Chapter 4.

- Chapter 5 will cover the discussion of the results and the conclusions of the project with recommendations for future work. 


\section{Multi-channel Alcoholic EEG Classification System}

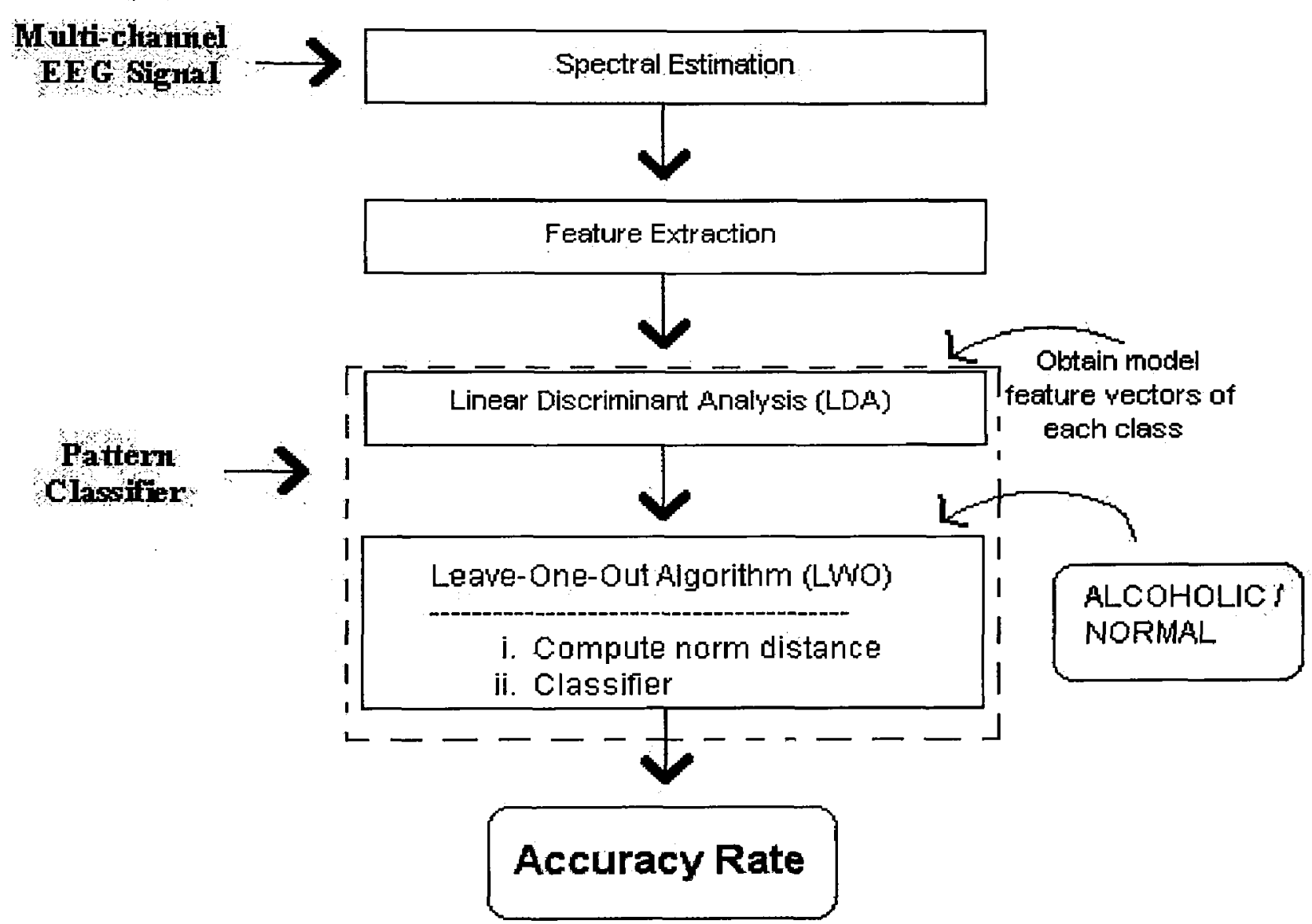

Figure 1.2: Flowchart of the proposed alcoholic screening system 


\section{Chapter 2}

\section{REVIEW}

This chapter presents a review of the research work done in this field including noninvasive diagnosis using EEG, Multi-channel DSP and EEG in alcoholism.

\subsection{NON-INVASIVE DIAGNOSIS USING EEG}

An EEG is usually measured as a continuous function of time which can be analyzed mathematically. In experimental EEG recording, it is often the changes of frequency content that are of interest. EEG signals can be categorized into broad or narrow frequency bands by the use of selective filters.

\subsubsection{EEG SIGNAL ANALYSIS}

Initially, the interpretation of EEG records is done by visual inspection and depends on recognizing patterns and their association with normal or pathological conditions. The widespread use of these methods is due to the relative technical ease with which the records can be made, the low cost of interpreting many records by visual inspection and the lack of information on the relative merits of other methods. But, 
these traditional methods have some disadvantages. Different diseases may give rise to apparently similar abnormalities and different interpretations may be made of the same record. It may be that the EEG does not contain any information which would improve its reliability and diagnostic value, but many alternative methods of deriving and analyzing EEG are being tried. One includes measurement and analysis techniques and associated developments in instrumentation. An analogue to digital conversion is done to analyze the signal using Computer. Digital computers are more efficient with a predetermined number of data values. In this case, EEG can be analyzed quantitatively in order to describe it numerically with improved reliability. The analysis can be based either on temporal or spatial localization.

The clinical use of the EEG depends on the recognition of particular waveforms having diagnostic significance, and the detection of focal EEG phenomena that have a relationship to focal pathology. The spatial distribution of EEG features over the surface of the head is thus of great importance. A number of techniques have been used for measuring and displaying the spatial characteristics. First, any of the methods already described for single-channel analysis is applied to each channel of a multi-channel record. The second method attempts to develop transformations of the EEG which indicate the site and distribution of abnormal patterns as contour maps. A feature of the spatial distribution of EEG signals is that when a waveform is present over a large area of the head, small time differences can occur at different sites. 


\subsubsection{SCREENING TECHNIQUES FOR ANY DISORDER}

Generally, there are three methods for seizure detection in EEG. First is a parametric approach based on a nonlinear estimation of model parameters for detection. The two other methods are nonparametric. The spectrum technique introduced by Gotman [9] uses frequency analysis to determine the changes in the dominant peak of the frequency spectrum of short epochs of EEG data. The autocorrelation technique introduced by Liu [11] performs analysis in the time domain using short epochs of EEG data. All these techniques are based on the assumption that the EEG signals are stationary or as a minimum locally stationary. Some methods Of processing EEG signals are given below.

\subsubsection{HJORTH ANALYSIS}

The quantitative description of EEG signals has been classified by Hjorth (1970) [4] into two types: (a) to define descriptive qualities for the general characterization of an amplitude / time pattern, e.g. the application of mathematical-statistical methods which also include frequency considerations; (b) to describe individual patterns or to detect specific patterns which can be defined in advance.

Hjorth analysis - Hjorth (1970) has developed a method of EEG-signal analysis which he would put in his type (a) class. Three parameters of an EEG signal are measured for successive epochs of one to several seconds duration. Two of these parameters are obtained from the first and second time derivatives of the amplitude fluctuations in the signal. The three parameters used by Hjorth are as follows. The first is called 'activity' and is the variance of the amplitude fluctuations in the epoch. 
The second is called 'mobility'. The third parameter is the 'complexity'. It is the ratio of the mobility of the first derivative of the signal to the mobility of the signal itself. Thus, for each analysis epoch three numbers are obtained that are representative of the pattern. Hjorth shows that these parameters describe the signal wave shape and are mathematically related to the power spectrum of the signal. The value of the Hjorth parameters is reduced if the signals have more than one major peak in the power spectrum. Despite this and the above limitations, they represent an important approach to the problem of quantifying the EEG with a small number of descriptors.

\subsubsection{AUTO-REGRESSIVE ANALYSIS}

The EEG is an unpredictable signal and it may have to be described by statistical parameters such as the mean and variance of the frequency spectrum from epoch to epoch. No matter how much is known about how the signal has fluctuated in amplitude up to the present instant of time. Another feature of this type of signal is that particular measurement will not be the same from epoch to epoch, even when recording conditions, the state of the subject are constant. As these features are a function of the process generating the signal, it may be more appropriate to derive a model of a generator that could produce them rather than describe the signal itself. The output of such a generator must have the same statistical properties as the signal, although the waveform generated by the model may look considerably different from the signal from which the model is derived. Two models are often used (Hurwicz, 1962). The first is called additive because it assumes that the randomness in the signal results from the addition of random amplitude values to a periodic fluctuation. The signal will have a constant-amplitude periodic fluctuation. The signal will have 
a constant-amplitude periodic auto-correlation function. The second is called autoregressive. The amplitude value at any time instant has two parts or components. One is correlated with all past values while the other is an added random perturbation. As a result of this, the signal will have an exponentially decaying periodic autocorrelation function (Dawson, 1953). It was found that EEG signals are more usually represented by the latter model than by the former. In the additive process, the time of occurrence of peaks and troughs of the signal can be at the time determined by the periodic component. Thus a disturbance changing the time of one peak will not affect the time of the next one. In the case of the auto-regressive model, a disturbance which shifts the time of a peak or trough alters the timing of all subsequent waves. The result of averaging would be that successive epochs tend to cancel each other and the average becomes more random and smaller in amplitude as the number of epochs is increased. This is what happens with EEG signals. The auto-regressive model is described in detail in the next chapter.

\subsubsection{SPECTRAL PARAMETER ANALYSIS(SPA)}

As the EEG signals can be approximated by an auto-regressive model, they may be simulated by passing random noise signals through a filter. The resultant 'EEG' depends on the characteristics of the filter. Therefore, the spectral analysis of the signal would result in a spectrum with a very similar shape to that of the filter characteristics and could thus be defined by a few filter parameters. This method is referred to as spectral parameter analysis.

The ultimate aim of many recent developments in EEG signal analysis is to make 
the whole procedure of analysis and interpretation automatic, with a computer generated report as the end result. Spectral analysis and other methods mentioned earlier enable the primary EEG signals to be quantitatively described with considerable reliability. In the further processing and interpretation of these data which present the real problem to the realization of the above aim. The problem could be to solve different brain diseases like Epilepsy, Newborn Seizure Detection, Sleep disorder, Meningitis, Schizophrenia, Trauma, Alcoholism.

\subsection{EEG IN ALCOHOLISM}

The communication between neurons creates electrical activity. A group of neurons with similar functions extend from one brain region to another, forming neural circuits. Circuits interact with one another to integrate the functions of the brain, including complex emotional, cognitive, and motivational processes. As pointed out, alcohol is a central nervous system depressant and it depresses the brain area most highly used in complex and rational thinking and voluntary control of behavior.

EEG is a rapid, inexpensive and non-invasive measure of relapse potential to alcohol in brain. Researchers have identified at least three brain wave patterns with EEG that have been associated with alcoholism and which may help to identify people at increased risk. Scientists have speculated that alcoholics and their offspring hold heightened beta EEG activity. Thus EEG signals can be successfully classified by analyzing the various developed signal processing techniques to detect alcoholism. 


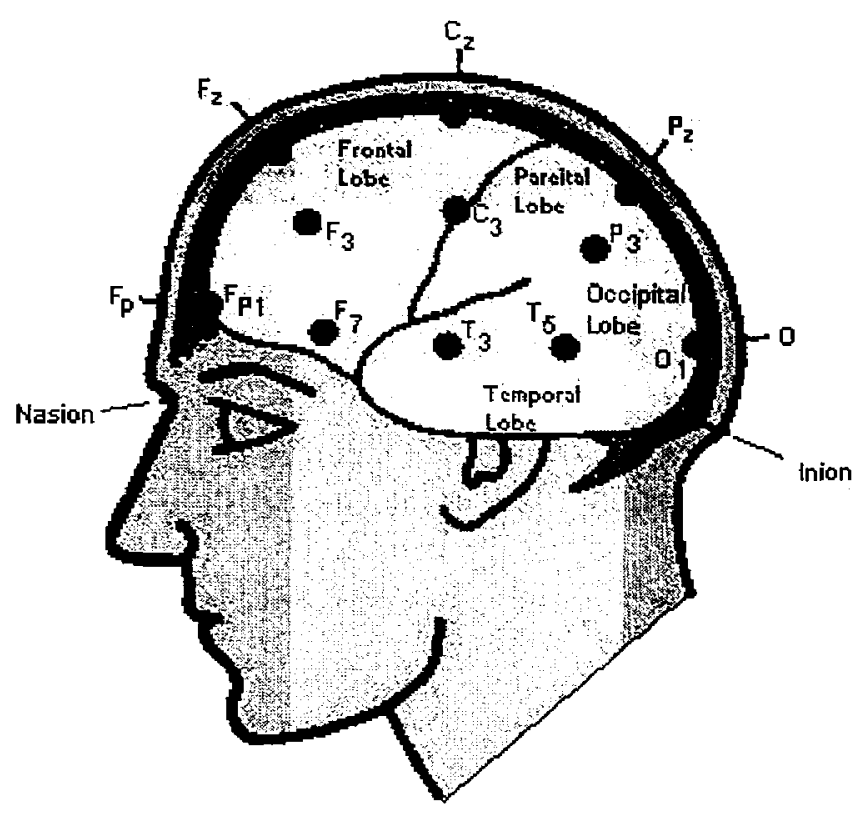

Figure 2.1: EEG Acquisition

\subsection{MULTI-CHANNEL ANALYSIS OF EEG}

An EEG signal consists of many channels that are captured using electrodes connected to the different parts of the brain. The multi-channel analysis enables EEGs to be compared on the basis of parameters from several channels simultaneously instead of just one. This could preserve the spatial information of the signal.

Normally, each signal consists of 32 channels coming from 32 electrodes connected to the different parts. Each electrode is labeled by giving a code consisting of alphar bets and numbers. The list of 32 electrodes with the corresponding labels is shown in the table below.

There are a few things to look for regarding the labeling of the electrodes. First, the electrode labels correspond to the respective location of the electrodes on the 


\begin{tabular}{|c|c|}
\hline List of electrodes used & Label \\
\hline 1 & FP1 \\
2 & FP2 \\
3 & AF3 \\
4 & AF4 \\
5 & F5 \\
6 & F3 \\
7 & F1 \\
8 & Fz \\
9 & F2 \\
10 & F4 \\
11 & F6 \\
12 & T1 \\
13 & FCz \\
14 & T2 \\
15 & T3 \\
16 & C5 \\
17 & C3 \\
18 & C1 \\
19 & Cz \\
20 & C2 \\
21 & C4 \\
22 & C6 \\
23 & T4 \\
24 & T5 \\
25 & P3 \\
26 & Pz \\
27 & P4 \\
28 & T6 \\
29 & O1 \\
30 & O2 \\
31 & EOGh \\
32 & EOGv \\
\hline
\end{tabular}

Table 2.1: Electrode Placement 
human scalp. Alphabets correspond to different areas of the skull, namely, $F$ for Frontal, $\mathrm{P}$ for Parietal, T for Temporal, O for Occipital, and $\mathrm{C}$ for Central and these locations are supposed to tap the EEG activity underneath those areas. Numbers attached with all the alphabets correspond to the sides of the scalp and ' $z$ ' as in case of $\mathrm{Fz}, \mathrm{Cz}, \mathrm{Pz}$ corresponds to the midline from frontal pole of the skull (just above eyes where nose joins the forehead) to the inion (where skull ends at the back of the head and can feel it by touching) where the hard and curved bone ends. Smaller number corresponds to its relatively nearer location to the midline, i.e., respective lobes $\mathrm{z}(\mathrm{Fz}$ etc. ) in comparison to the larger number whereas odd number means left side of the skull and even number means right side of the skull. The labeling is shown in Figure 2.1 .

For example, 'Fz' means Frontal midline, F1 means immediate left side electrode location from the midline, and F2 means immediate right side electrode location from the midline. As the number increases, those locations will go farther away from 'Fz'. FP corresponds to the Frontal Pole. There are different systems of fixing the locations. The international accepted way of electrode placement is $10 / 20$ system proposed by Jasper (1958) [2]. The 10 and 20 correspond to the percentage of the distances of the electrodes on the scalp. For instance, $10 \%$ is the percentage of total length of an imaginary line for Frontal locations from left to right (ear to ear). 20\% is the percentage of total length of and imaginary line for not only midline but for other arrays of electrode locations from front to back. All this percentage is calculated on the basis of the number of electrodes one uses.

Two channels, FP1 and F3 taken from the frontal lobe of the scalp are shown in Figure 2.2. 

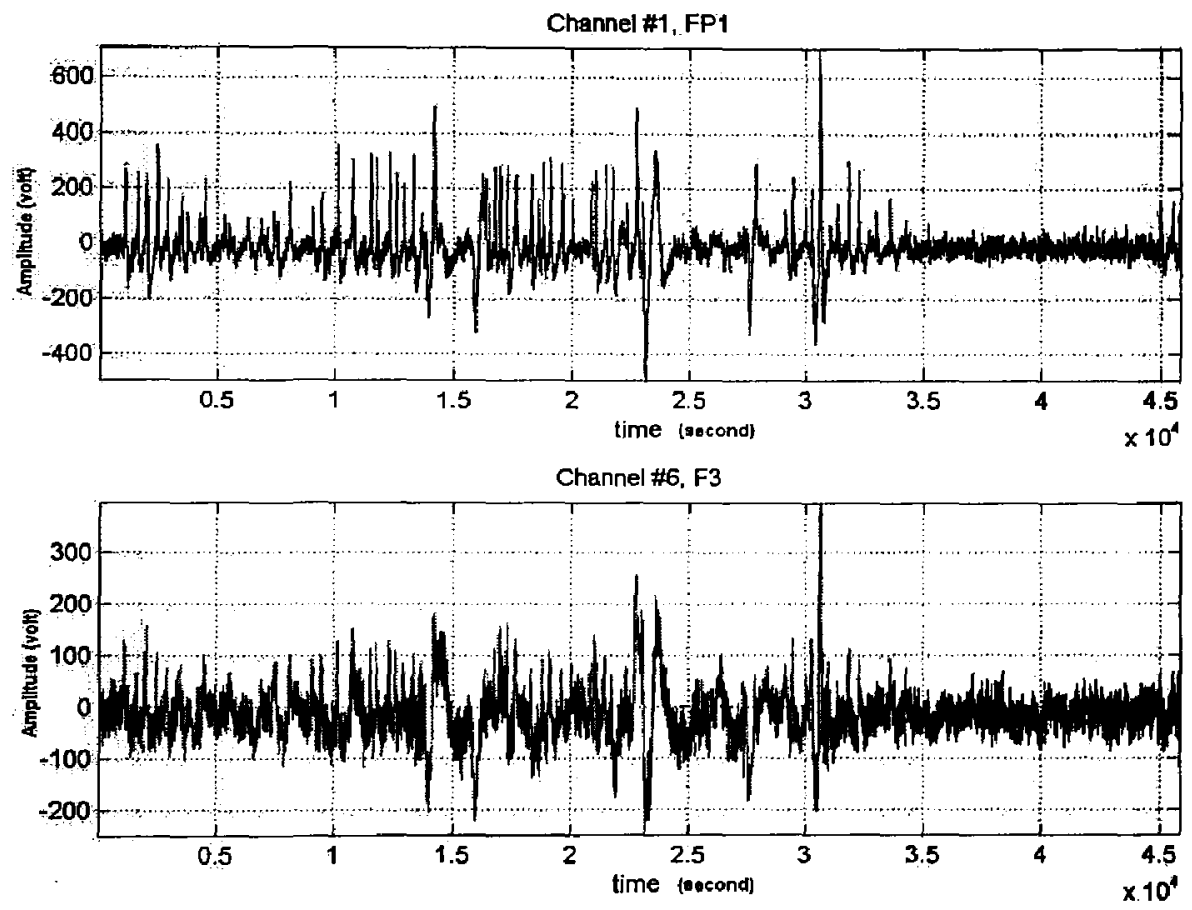

Figure 2.2: Different channels of an EEG signal 
Therefore, according to table 2.1 the first 30 channels are attached to the electrodes in different locations and the last two channels (i.e. 31 and 32) are used for eye movement recordings for Horizontal (31) and Vertical (32) eye movements for doing correction.

Research has indicated that better and reliable differentiation is given by the channels which are more closer to the center than to the periphery. It has also pointed out that the eye movement recordings are crucial in detecting alcoholism. The eye movement recordings of alcoholic patients show a major variation compared to normal ones. With respect to Table 2.1, the channels concentrated in the center area are $\mathrm{Fz}, \mathrm{F} 3, \mathrm{~F} 4, \mathrm{Cz}, \mathrm{C} 3, \mathrm{C} 4, \mathrm{Pz}, \mathrm{P} 3, \mathrm{P} 4, \mathrm{O} 1$ and $\mathrm{O} 2$. Hence, these channels are given a closer examination while detecting alcoholic EEG signals.

In multi-channel analysis, all associated channels can be exploited simultaneously to extract information of interest as shown in figure 2.3.

\subsubsection{MULTICOMPONENT CHARACTERISTICS OF EEG}

To take multicomponent features into account, both autocorrelation and spectrum techniques must be extended by including both time variation and frequency variation parameters in the basic equations on which these techniques are based. The extension of these methods leads to a time-frequency formulation in the $(t-f)$ domain or a time-varying autocorrelation formulation in the time-lag domain. Hence the multicomponent aspect requires a selection of an appropriate time-frequency distribution that is capable of handling multicomponent signals. Then a calibration is needed to translate the detection criteria from the spectrum and autocorrelation domain to the 


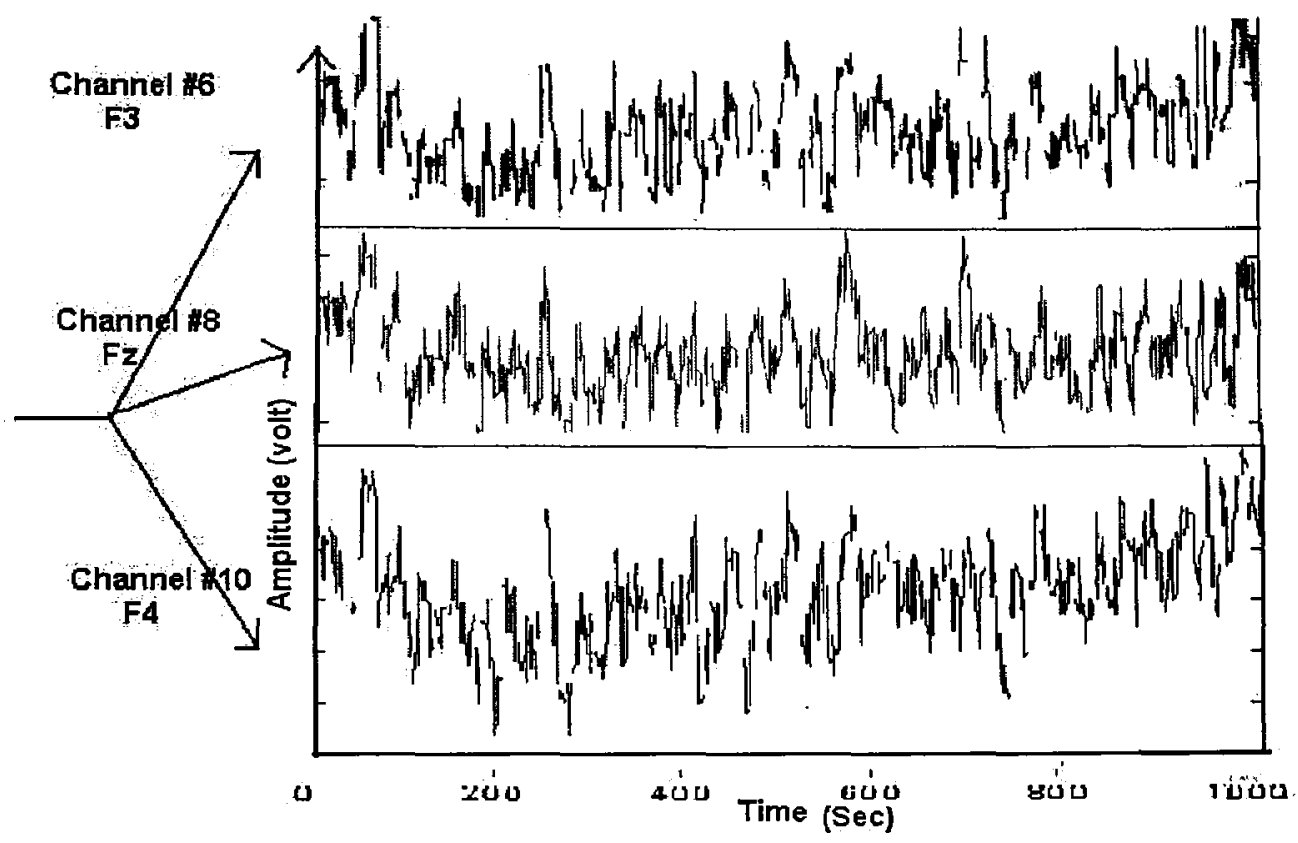

Figure 2.3: Multichannel EEG signals

time-frequency domain. 


\section{Chapter 3}

\section{METHODOLOGY}

This chapter explains the theory of the project including multi-channel AR modeling, Cepstral analysis and other techniques involved in the parametric extraction of the signal. The feature extraction in this project is based on AR modeling and Cepstral theory.

\subsection{SEGMENTATION OF EEG}

An EEG is described as non-stationary time series because the pattern of fluctuation of one epoch never exactly repeats itself. The signal is also non-linear and in order to apply one of the existing compression techniques, the signal is assumed to be linear. In order to make a non-stationary signal appear stationary, the signal is segmented. In other words, since the spectral composition of EEG signal changes with time, a finite-duration segment is used to compute the spectrum of the signal. The signal is segmented using fixed window, specifically Hanning window is used in this project.

The process of sampling a time varying function for a finite amount of time is 


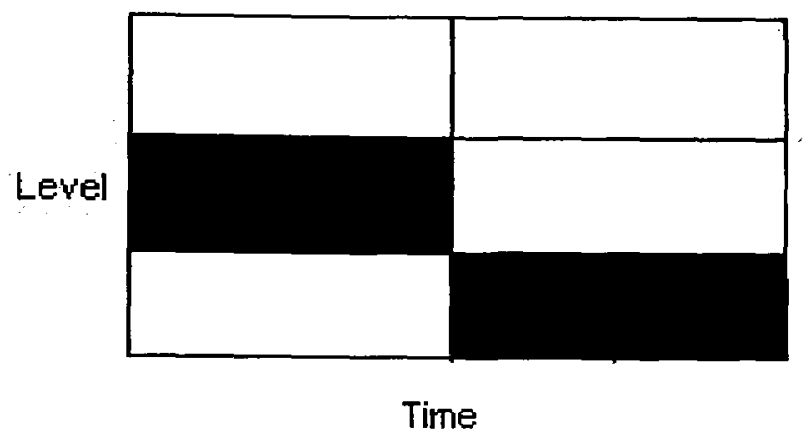

Figure 3.1: Segmentation Chart

equivalent to multiplying the function with a window. The desirable window characteristics are as follows:

- Small width of main lobe of frequency response of the window containing as much of the total energy as possible.

- Side lobes of the frequency response that decrease in energy rapidly as $\omega$ tends to $\pi$.

In order to extend this to multivariate data and estimate coherencies between channels of EEG signals, optimal segmentation should be determined. One way to obtain the best segmentation is by dividing the time series into blocks in a dyadic manner as shown in figure 3.1, with a minimum objective cost function. This helps to determine the stationarity of the signal.

Adaptive segmentation is another approach to this problem. The method requires a procedure for testing for non-stationarities for detecting the boundaries of segments 
during which parameters defining stationarity are stable and a means of classifying the types of signal that occur during the detected segments. It is very close to quantifying the procedure of visual analysis of the EEG.

\subsection{SPECTRAL ESTIMATION OF THE SIGNAL}

Spectral analysis is a fundamental tool to study the time varying activity of EEG. After segmentation, each segment is approximated to be stationary. Now the most common way to find the spectral analysis is by using Fourier transform. Since it is perfectly localized in frequency and are periodic, the Fourier functions are ideal for stationary signals. It can be estimated by computing the periodogram which are simply the square of the modulus of the Fourier coefficients.

Thus Fig. 3.2 shows the transformation of an alcoholic EEG signal computed using FFT for a segment of channel 1. Then the next figure is an estimate of the spectrum computed using periodogram for the same part of the channel as above.

The Z- transform is a unique representation of a sequence in the complex $\mathrm{Z}$ plane. A typical method of displaying the information that the Z-transform contains is in terms of poles and zeros of the sequence. The poles and zeros of the Z- transform are plotted using a pole-zero map. Pole Zero plot allows gathering information about the designed model used for feature extraction and spectral evaluation of the signal. Poles are defined as the complex frequencies that make the overall gain of the filter transfer function infinite. Zeros are complex frequencies that make the overall gain of the transfer function zero. The angle $2 \pi$ of the pole-zero plot will be the sampling 


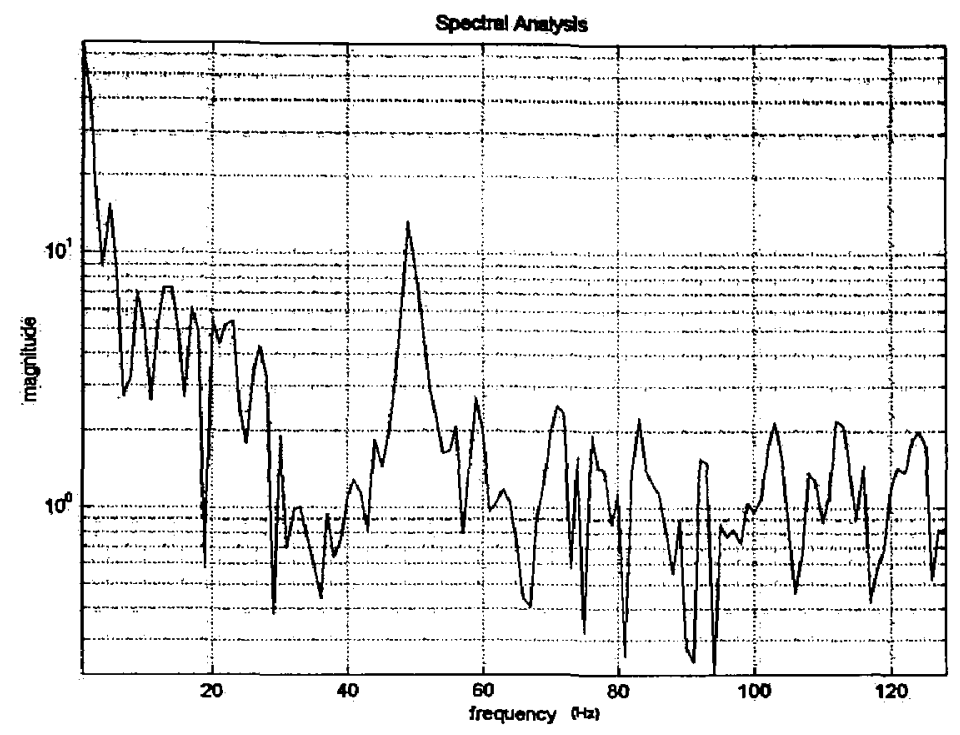

Figure 3.2: Transformation of alcoholic EEG segment using FFT

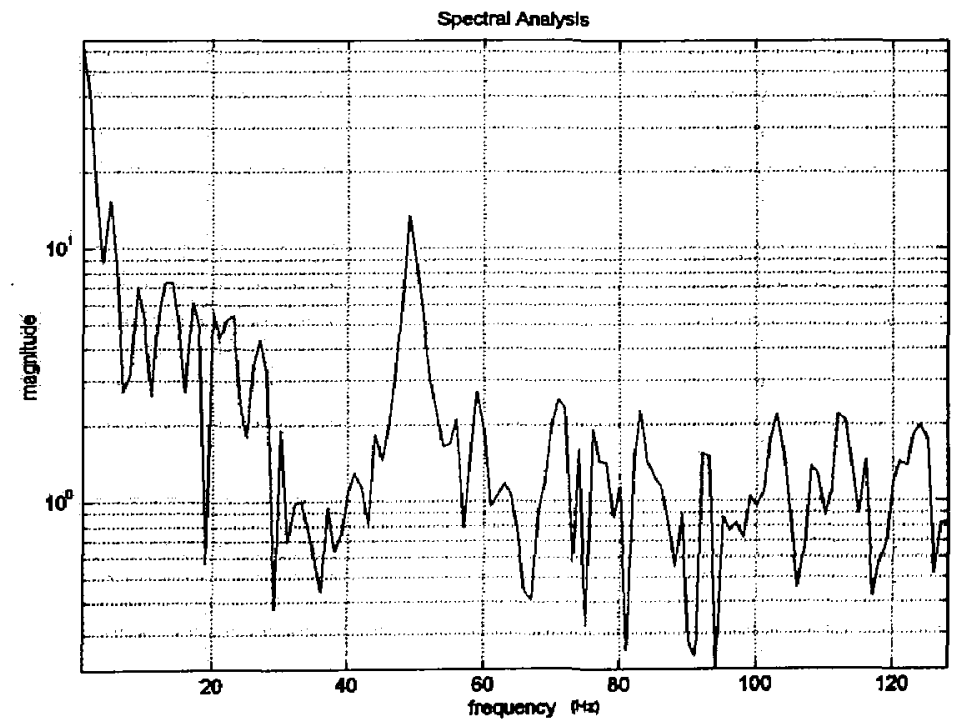

Figure 3.3: Periodogram 


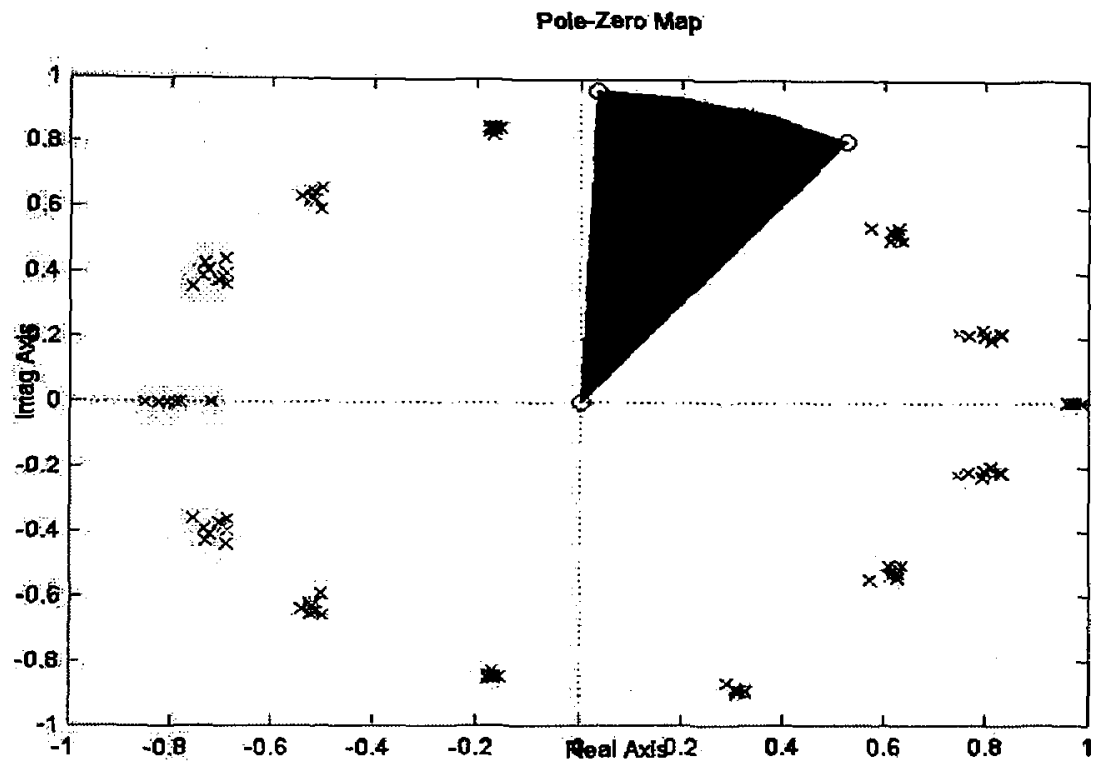

Figure 3.4: Pole-zero plot

frequency of the signal. The radius of pole-zero plot is an index of the spectral peak dominancy and the poles closer to the unit circle represent highly dominant spectral peaks. There are two regions of convergence for the poles of the system. If the region of convergence includes the unit circle, then the system being represented is stable.

For example, the pole-map of an alcoholic signal after fixed segmentation of all channels into 10 segments based on temporal distribution is shown in figure 3.4. The shaded region in the pole-zero plot identifies the frequency range of $41 \mathrm{~Hz}$ to $63 \mathrm{~Hz}$ 
and radius up to 0.965 . Based on an earlier temporal based analysis, poles tend to appear in the shaded portion of the figure for alcoholic signals.

\subsection{DESCRIPTION OF AR MODEL}

The EEG generating process can be modeled by an autoregressive process for spectral estimation. It attempts to define EEG signals in terms of a small number of parameters. Therefore, this can be used for compression of signals. An auto-regressive (AR) model is a commonly used tool in DSP (due to its low computational time cost) to model stationary signals as all-pole functions whose transfer function is:

$$
H(z)=\frac{1}{1+\sum_{i=1}^{M} a_{i} z^{i}}
$$

Where $\mathrm{H}(\mathrm{z})$ is the $\mathrm{z}$ transform of the signal, $a_{i}$ are auto regressive coefficients, and $\mathrm{M}$ is the filter order. This expression can also be explained in terms of the poles once the denominator is factorized. So, equation (3.3.1) can be re-written as:

$$
H(z)=\frac{1}{\left(z-p_{1}\right)\left(z-p_{2}\right) \ldots\left(z-p_{n}\right)}
$$

With the appropriate number of poles, the signal can be reconstructed with a finite, and in some cases, with a small number of coefficients. That makes the AR modeling useful for applications that do not need a high degree of accuracy in terms of signal reconstruction. Some of the common ways to estimate the AR parameters or 
the prediction coefficients are the autocorrelation, covariance method and the YuleWalker method.

In the autoregressive model, the signal at a particular instant is expressed as a weighted sum of the values at preceding instants together with a random component. The weighting values are the parameters of the model, and the number of preceding instants used defines its order. The model order is an important parameter in order to extract all the useful properties of the digitalized signal.

There is not yet a satisfactory method available for a clear determination of the optimal order of an auto-regressive model. However, in this project the approximate model order is determined by calculating the approximate number of peaks above a certain threshold in the frequency domain of the signal.

Linear time series analysis tools such as linear prediction is used for extracting the features in the AR model. Hence, it compresses the data without losing much clinical information. Thus the non-stationary signals are analyzed using segmentation and linear prediction.

\section{Multi-channel AR Modeling}

AR modeling based on single channel analysis can be used to analyze only the information from a single part of the scalp. But it may also require information from the other channels at the same time for improving the accuracy of the analysis. Therefore, multi-channel AR modeling is a better approach to this problem.

The technique of multi-channel AR modeling was developed by Nuttall as an extension of single-channel Burg's algorithm. In this technique, the multi-channel linear prediction coefficients are estimated by solving the normal equation 


$$
\left[\begin{array}{ccccc}
I & A_{l}^{p} & \ldots \ldots & A_{p-l}^{p} & A_{p}^{p} \\
B_{p}^{p} & B_{p-l}^{p} & \ldots \ldots & B_{l}^{p} & I
\end{array}\right]\left[\begin{array}{cccc}
R_{0} & R_{1}^{H} & \ldots \ldots & R_{p}^{H} \\
R_{1} & R_{0} & \ldots \ldots & R_{p-1}^{H} \\
\cdot & . & \ldots \ldots & \cdot \\
\cdot & . & \ldots \ldots & . \\
\cdot & \cdot & \ldots \ldots & . \\
\cdot & . & \ldots \ldots & . \\
R_{p} & R_{p-1} & \ldots \ldots & R_{0}
\end{array}\right]=\left[\begin{array}{cccc}
U_{p} & 0 & \ldots \ldots & 0 \\
0 & 0 & \ldots \ldots & V_{p}
\end{array}\right]
$$

where $R_{i}$ is the covariance function and is defined as

$$
R_{k}=E\left[X_{n+k} X_{n}^{H}\right]
$$

$U_{p}$ is the forward prediction error covariance and $V_{p}$ is the backward prediction error covariance, and $A_{i}^{p}$ and $B_{i}^{p}$ are the forward and backward linear prediction coefficient matrices, respectively. The normal equation can be solved by using multichannel Levinson-Wiggins Robinson or multi-channel Levinson-Durbin algorithm to obtain the coefficient matrices. The existing Multi-channel linear predictive coding algorithm calculates the coefficients for each channel recursively and constructs a matrix. The matrix contains redundant information. In order to avoid the abovementioned problems, an algorithm should be developed that would only take the average of the coefficients of the channels that are similar or correlated. Therefore, the amount of redundant information is reduced while retaining the spatial information. 


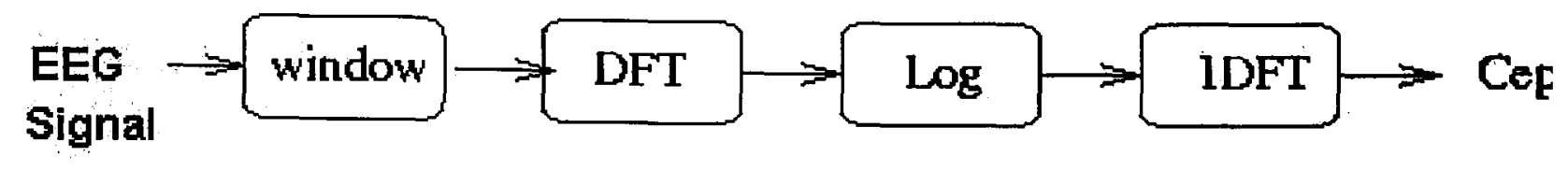

Figure 3.5: Cepstral Analysis

\subsection{CEPTSTRAL THEORY}

Cepstral theory is applied to the EEG signals since it is widely used in periodic signals for spectral evaluation. The steps involved in forming the cepstrum from an EEG signal is shown diagrammatically in figure 3.5 .

Cepstral analysis provides a means of determining the poles, zeros, and high frequency gain of the modeling filter which will help the spectral estimation of the signal. The word cepstrum was coined by reversing the first syllable in the word spectrum. The cepstrum is a decaying function of time which exists in a domain referred to as quefrency (reversal of the first syllable in frequency), which has units of time [7].

The complex cepstrum of a data sequence is defined as the inverse Z-transform of the complex logarithm of the Z-transform of the data sequence, i.e.

$$
\frac{1}{2 \pi j} \oint_{c} \log (X(z)) z^{n-1} d z
$$

The cepstrum of a signal is defined as the inverse Fourier transform of the log power spectrum of the signal. If the signal is stable (all the coefficients are inside the unit circle, in the complex Z-plane, the logarithmic transfer function $\ln (\mathrm{H}(\mathrm{z}))$ can be expressed as: 


$$
\ln H(z)=c(z)=\sum_{i=1}^{M} c_{i} z^{-i}
$$

There is a non-linear relation between the AR coefficients [3] that links the AR coefficients with the cepstral coefficients. That is:

$$
\begin{gathered}
c_{1}=-a_{1}, i=1, \\
c_{i}=-a_{i}-\sum_{i=1}^{i-1}\left(1-\frac{k}{i}\right) a_{k} c_{i-k}, 1<i \leq M
\end{gathered}
$$

Fourier Transform is the natural way to separate the transfer function $\mathrm{H}(\mathrm{z})$ and the excitation component $\mathrm{E}(\mathrm{z})$ of the signal. This produces the cepstral analysis. In figure 3.5 , a segment of a signal is extracted with a hamming window. The application of the Hamming window reduces discontinuity. For one segment of a channel of alcoholic EEG, the resulting (real) cepstral analysis is shown in figure 3.6. Usually, most of the detail occurs near the origin and in peaks higher up the cepstrum. Thus the lower numbered coefficients provide the envelope information. The remainder of the detail is mostly contained in the peaks which are separated by the pitch period and provide the fine detail pitch information.

Also, the determination of the relative positions of two signals on the time axis with the help of cepstral analysis is characterized by higher immunity to signal fluctuations than a direct measurement of their positions. The cepstrum is also widely used in speech processing to deconvolve the periodic voiced excitation signal from the effects of the vocal tract. 


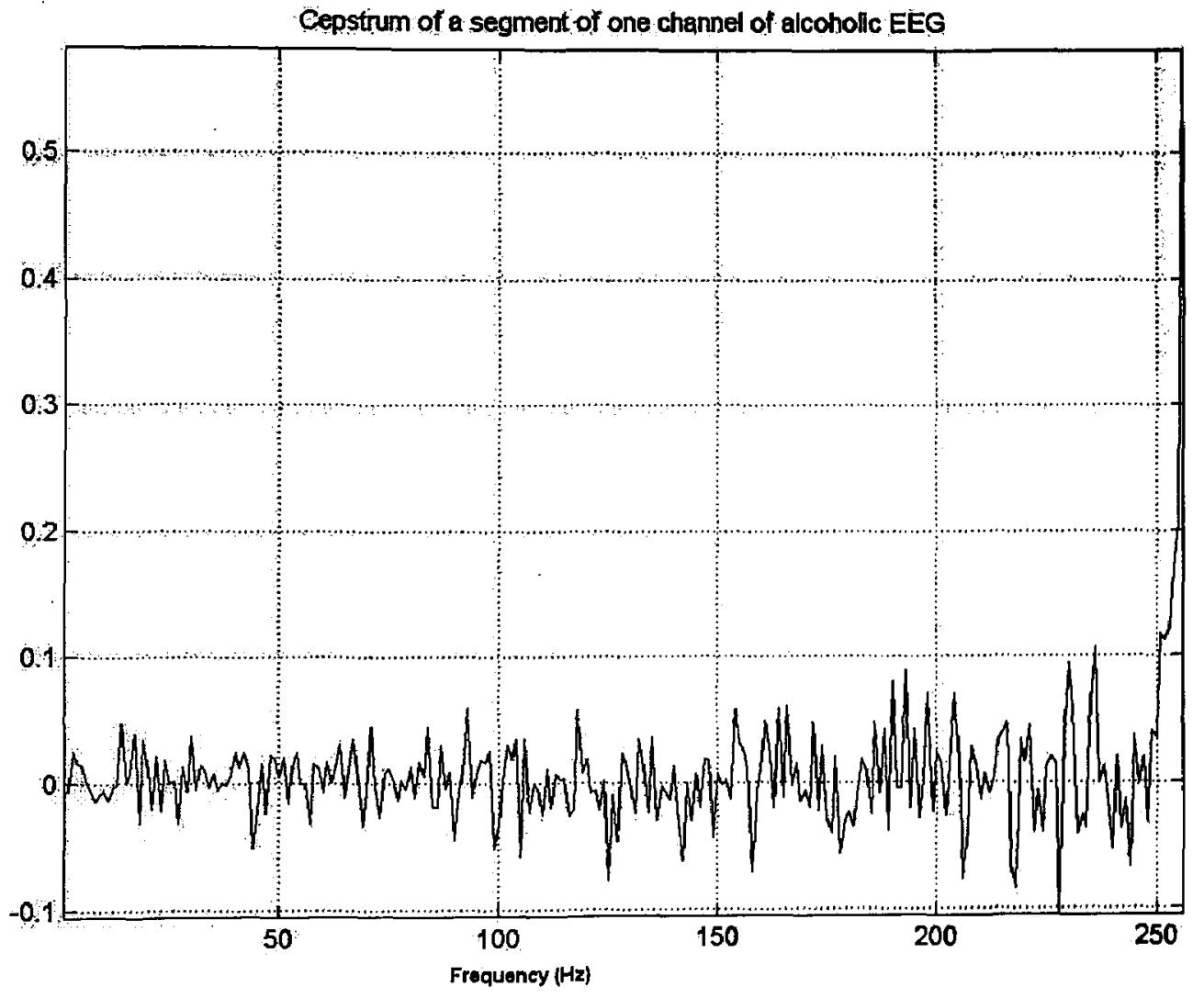

Figure 3.6: Real Ceptrum 
$\mathrm{AR}$ and cepstral coefficients can be used to extract features of the signal and the signals can be reconstructed using these few coefficients. One important factor in extracting signal features is to select the maximum amount of information useful for discriminating the main properties of the signal with the minimum number of coefficients. The model order is also an important parameter in order to extract all the useful properties of the signal.

To summarize, this chapter achieved data reduction by selecting only the central channels of the EEG which contains the core information. Following that, a fixed segmentation was applied on those channels. Then by applying cepstral analysis and AR modeling on the segments, feature extraction was attained. This process was attained by using the MATLAB software.The classification of EEG patterns based on these features is the next step in the project. 


\section{Chapter 4}

\section{PATTERN CLASSIFICATION}

It is apparent that reliable interpretation of the EEG requires detection and discrimination between different waveforms or wave patterns. In the case of EEG, methods for detecting specific patterns have been developed largely on an empirical or intuitive basis. The information necessary to recognize the patterns is derived using feature extraction. The information must then be processed in some way so that the pattern of features correlating with particular disease states can be determined. In this work, pattern classification was applied to distinguish between the normal and alcoholic signals. This chapter presents the theory involved in the pattern classification of the multi-channel analysis such as Linear Discriminant Analysis, Leave-One-Out method in the project.

\subsection{LINEAR DISCRIMINANT ANALYSIS (LDA)}

One way to decide the features associated with a particular disease state is to use a statistical technique called linear discriminant analysis [5]. Discriminant analysis is a method for distinguishing patterns containing many features by maximizing the ratio of between the class variance to the within class variance in any particular data set, 
thereby guaranteeing maximum seperability. This can be used for multivariate data analysis.

In this project, Alcoholic and Normal signals are separated as two classes. LDA performs a dimensionality reduction while preserving as much of the class discriminatory information as possible. It computes a class-dependent and class-independent based transformation. Class-dependent LDA produces one transformation per class to discriminate that class from all other classes. Class-independent LDA produces only one transformation to maximally separate all classes. Thus, LDA creates feature vector based on each class model, i.e. for alcoholic and normal.

\subsection{LEAVE ONE OUT ALGORITHM (LWO)}

The accuracy rate of the system, and the selection of the reference vector are performed using the Leave One Out (LWO) method, which extracts in each stage of the loop one reference signal based on each of alcoholic and normal subject model to train the system and leave the remaining as reference vectors.

Then, the objective is to extract and compare the distance between the input pattern vector and all the reference vectors. There are several distances that can be used. The normal Euclidean distance, the Mahalanobis distance and the maximum likelihood measurement (MLM) are some of them [6].

The Maximum Likelihood Method (MLM) (or Euclidean distance) is used to extract the norm that calculates the distance between the signal under test and the reference vector.

The spectral estimates obtained by MLM and AR signal modeling are related. 
The MLM is the reciprocal of the sum of all $N$ number of AR spectral estimates. The performance of the MLM is determined by both the mean and variance vectors of the feature. The performance of the MLM becomes poorer as features with high variability increase. The MLM distance most closely approximates the optimal Bayes

classifier and obtains the best discriminative efficiency among the three proposed classifiers, if the probability density functions of the features extracted are multivariate Gaussian. Let $X$ be the random variable that refers the feature extracted according to one of the above feature extraction methods. Assuming that the Probability Density Function (PDF), $P\left(X \mid w_{i}\right)$, is multivariate Gaussian:

$$
P\left(X \mid w_{i}\right)=\frac{1}{(2 \pi)^{\frac{N}{2}}\left|V_{i}\right|} \exp \left[-\frac{1}{2}\left(X-M_{i}\right)^{\prime} V_{i}^{-1}\left(X-M_{i}\right)\right]
$$

Where $V$ is the covariance matrix and $M$ is the average matrix of the process.

The discriminant function becomes:

$$
d_{j}(X)=-\frac{N}{2} \ln (2 \pi)-\frac{1}{2} \ln \left|V_{i}\right|-\frac{1}{2}\left[\left(X-M_{j}\right)^{\prime} V_{j}^{-1}\left(X-M_{j}\right)\right]
$$

For all $j$ the first term is the same, so it can be dropped from the study, so:

$$
d_{j}(X)=\frac{1}{2} \ln \left|V_{i}\right|+\frac{1}{2}\left[\left(X-M_{j}\right)^{\prime} V_{j}^{-1}\left(X-M_{j}\right)\right]
$$

which is the distance between input samples and references vectors that has been used in the experiments. 
This distance will be used to estimate how far from the reference groups each sample signal is located. The procedure that has been followed using the "leave one out" algorithm (LWO) is to use the same sample signal under test as reference vector. This algorithm is repeated for the entire training database.

That is, LWO excludes each signal from the reference vectors in each turn. Then during each turn, the Maximum Likelihood Method again computes the distance or norm between the signals under test and some reference vectors selected previously with the LWO method. So, LWO method maintains independence between the results and the data set, since each signal is excluded from the reference vectors in each turn.

To summarize, the feature extracted from all the signals are included in the database and have to reference vectors as "normal" or "abnormal" signal. When a test signal is extracted from the reference database, the MLM or Euclidean distance of the test signal to the reference groups is computed. This operation is repeated for all the signals in the database. If the distance of the test signal to the group it originated is less than the other group, it means that it has been successfully classified. The purpose of this method is to obtain the overall accuracy rate of the classifier.

\subsubsection{PATTERN ANALYSIS OF EEG SIGNAL}

Pattern classification was achieved by obtaining the necessary differentiation between the alcoholic and non-alcoholic EEG signal features after the application of Linear Discriminant Analysis using Leave One Out Classification technique. LDA extracted the feature vectors based on the norm distances calculated using MLM and Euclidean distance. Finally, using the statistical based LWO method the signal features were successfully classified. This process was implemented using a statistical software 
package called SPSS. The detailed description of the results from the classifier are given in the next chapter. 


\section{Chapter 5}

\section{RESULTS AND DISCUSSION}

This chapter presents the results obtained by the multi-channel analysis based on the model used.

\subsection{RESULTS USING FEATURE ANALYSIS}

The alcoholic EEG signals used in the project include 32 channels recorded from different portions of the brain for three minutes duration. The sampling frequency of the signal is $256 \mathrm{~Hz} .25$ signals of each subject (Normal and Alcoholic) were taken for analysis. The core channels (Fz, F3, F4, Cz, C3, C4, Pz, P3, P4, O1 and O2) which are closer to the center were considered for the multi-channel analysis. Eye movements recordings (channel \# 31 and 32) were also inspected simultaneously. Spectral estimation of these channels show distinct changes.

For example, the variation in the horizontal eye movement recordings between the alcoholic and normal EEG signal can be seen from Fig. 5.1. The alteration in one of the central channels of the alcoholic and normal signal is visible in Fig. 5.2. 

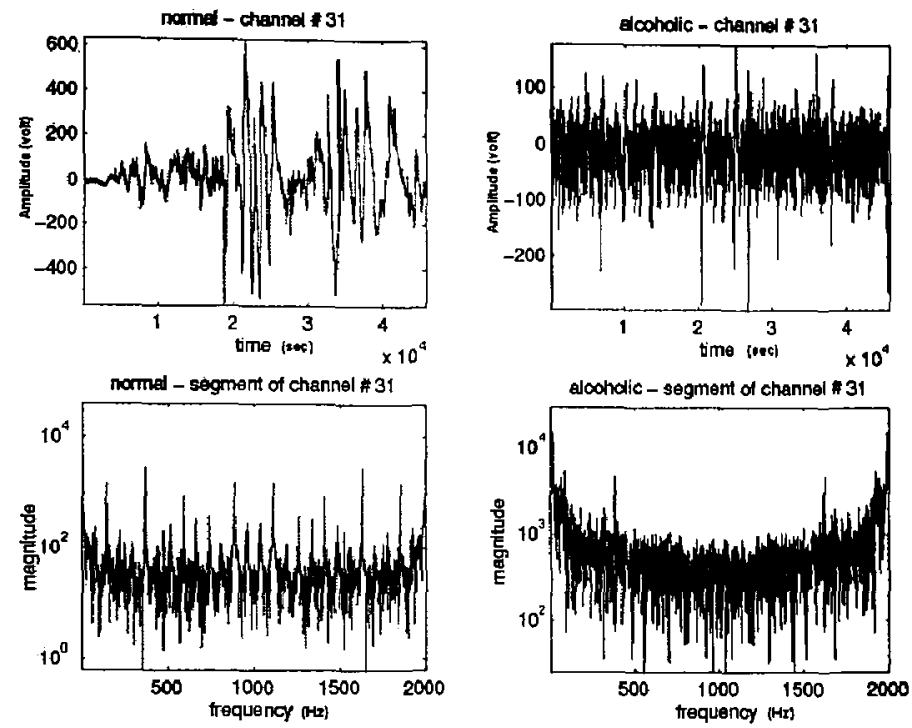

Figure 5.1: Periodogram
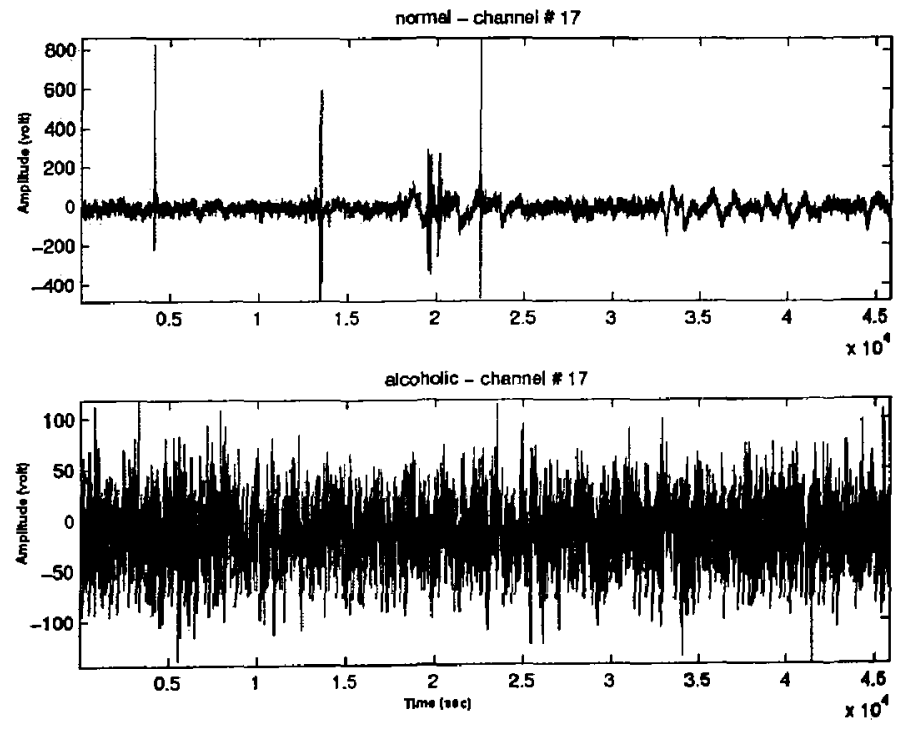

Figure 5.2: EEG channel 17 


\section{CLASSIFICATION STATISTICS}

\begin{tabular}{|lll|}
\hline \multicolumn{2}{c}{ Classification Processing Summary } \\
\hline Excluded & \multicolumn{1}{c|}{$\begin{array}{l}\text { Missing or out-of-range } \\
\text { group codes }\end{array}$} & 50 \\
& $\begin{array}{l}\text { At least one missing } \\
\text { discrimination variable }\end{array}$ & 0 \\
Used in Ouptput & & 50 \\
\hline
\end{tabular}

Figure 5.3: Classification Summary Results

Therefore, in this case 13 channels out of 32 were selected for comparison. Using AR modeling, signal features were extracted for applying the pattern classification techniques. The model order was taken as 10.

\subsection{RESULTS OF PATTERN CLASSIFICATION OF ALCOHOLIC EEG}

A classifier is developed based on Linear Discriminant Analysis and Leave-One-Out method for the alcoholic and normal signal features extracted. First, the feature vector was computed using the LDA method. Once the initial parameters of the feature extraction block were selected, a proper analysis was made using the LWO method by comparing the features extracted to the group of normal signals and the alcoholic groups. In other words, LWO excludes each signal from the reference vectors 
Classification Results

\begin{tabular}{|c|c|c|c|}
\hline \multirow[b]{2}{*}{ CAT } & \multicolumn{2}{|c|}{ Predicted Group Membership } & \multirow[t]{2}{*}{ Total } \\
\hline & $\mathrm{ALC}$ & NOR & \\
\hline Original Count & 25 & 0 & 25 \\
\hline Hormal & 0 & 25 & 25 \\
\hline Alcohol & 100.0 & .0 & 100.0 \\
\hline Normal & 0 & 100.0 & 100.0 \\
\hline Cross Y alidated Count ALC & 20 & 5 & 100.0 \\
\hline HOR & 9 & 16 & 100.0 \\
\hline ALC & 80.0 & 20.0 & 100.0 \\
\hline NOR & 36.0 & 64.0 & 100.0 \\
\hline
\end{tabular}

a. Cross validation is done only for those cases in the analysis. In cross validation, each case is classified by the functions derived from all cases other than that case.

b. $1000 \%$ of original grouped cases correctly classified.

c. $72.0 \%$ of cross-validated grouped cases correctly cl assified.

Figure 5.4: Classification Table with AR modeling 
Classification Results

\begin{tabular}{|c|c|c|c|}
\hline \multirow[b]{2}{*}{$\mathrm{CAT}$} & \multicolumn{2}{|c|}{ Predicted Group Membership } & \multirow[t]{2}{*}{ Toteit } \\
\hline & $\mathrm{ALC}$ & $\mathrm{NOR}$ & \\
\hline Original Count & 25 & 0 & 25 \\
\hline Notmal & 0 & 25 & 25 \\
\hline Alcohol & 100.0 & .0 & 100.0 \\
\hline Normal & 0 & 100.0 & 100.0 \\
\hline Cross V alidated Count ALC & 18 & 7 & 100.0 \\
\hline $\mathrm{HOR}$ & 12 & 13 & 100.0 \\
\hline ALC & 72.0 & 28.0 & 100.0 \\
\hline $\mathrm{HOR}$ & 48.0 & 52.0 & 100.0 \\
\hline
\end{tabular}

a. Cross validation is done only for those cases in the analysis. In cross validation, each case is classified by the functions derived from all cases other than that case.

b. $1000 \%$ of original grouped cases correctly classified.

c. $62.0 \%$ of cross-validated grouped cases correctly classified.

Figure 5.5: Classification Table with Cepstral Theory 
during each turn based on the distance or norm computed between the signals under test and some reference vectors using the Maximum Likelihood Method. This was repeated for the entire training database. Using LWO, the signal features based on AR were classified based on the norm distances to maximize the accuracy. The classifier thus obtained gave the overall accuracy rate of the system. The accuracy rate obtained is $72 \%$. The results obtained after the classification is shown in Figure 5.4. Then the Cepstral based accuracy rate was obtained as $62 \%$ and the tabulated results are shown in Figure 5.5.

The possibilities of this analysis are very high since the classifier extracts any features using the LWO method that make the difference between normal and abnormal cells. Thus, the results obtained in this phase of the project confirms the objective outlined earlier.

\subsection{DISCUSSIONS AND FUTURE WORK}

Using the statistical software package called SPSS, the signal features were successfully classified based on the LWO method with an accuracy rate of $72 \%$ with AR modeling and $62 \%$ with Cepstral theory for the cross-validated data set. This is acquired from a data set of 50 signals. The results could be improved by applying the process to a different set of data with a longer period. The original grouped cases were classified correctly as alcoholic and non-alcoholic with 100\% accuracy using both Cepstral and AR modeling. Overall, this is a powerful method that can extract information that is not apparent from single-channel temporal based analysis. The process in the project can extract information using multiple channel analysis with 
reasonable accuracy from channels closer to the center of the scalp and the eye movement recordings. This method preserves the spatial information and is encouraged to further explore to improve the precision. Another multichannel analysis technique called SLEX analysis could be combined with this to improve the accuracy of the classifier. 


\title{
Appendix A
}

\author{
Abbreviation
}

EEG Electroencephalograph

SPSS Statistical Software Package

LWO Leave-One-Out

LDA Linear Discriminant Analysis

SPA Spectral Parameter Analysis

AR Auto-Regressive

DSP Digital Signal Processing 


\section{Bibliography}

[1] Fay S. Tyner, John R. Knott and W. Brem Mayer. Fundamentals of EEG Technology Volume 1, Basic Concepts and Methods', New York: Raven Press, 1983.

[2] Paul B. Colditz, Chris J. Burke, Patrick Celka. Brain Storming, "Digital Processing of EEG Signals IEEE Engineering in Medicine and Biology Magazine, Volume 20.

[3] Emerging Medical Imaging Technologies and Data Acquisition and Modeling, IEEE Signal Processing Magazine, Volume 18.

[4] Fay S. Tyner, John R. Knott and W. Brem Mayer. Fundamentals of EEG Technology Volume 2, Basic Concepts and Methods', New York: Raven Press, 1983.

[5] Suresh Balakrishnama, Aravind Ganapathiraju, Joseph Picone. Linear Discriminant Analysis for Signal Processing Problems Institute of signal and In formation processing. , 1999 IEEE southeast contest, Mississipi State University. Mississippi, USA. Email: balakrish,ganapath, picone @isip.msstate.edu

[6] Lisa J. Lazareck and Zahra Moussavi Adaptive Swallowing Sound Segmentation by Variance Dimension Department of Electrical Engineering, University of Manitoba, Winnipeg, Canada, R3T 2N2 E-mail: lazareck@ee.umanitoba.ca, mousavi@ee.umanitoba.ca

[7] M. N. Ahmed, S. M. Yamany, A. A. Farag, and T. Moriarty Bias Field Estimation and Adaptive Segmentation of MRI Data Using a Modifed 
Fuzzy C-Means Algorithm Univ. of Louisville, E.E. Dept., Louisville, KY 40292 E-mail:fmohamed,faragg@cairo.spd.louisville.edu Department of Neurological Surgery, University of Louisville, Louisville, KY 40292

[8] R. Rangayyan and S. Krishnan, D. Bell, C. Frank, K. Ladly. Parametric Representation and Screening of Knee Joint Vibroarthrographic Signals . IEEE Transactions on Biomedical Engineering. Vol. 44 (11), pp 1068-1074 November 1997.

[9] The Data Analysis Brief Book, http://rkb.home.ch/rkb/littleA.html

[10] North American Congress on Biomechanics, University of Waterloo, Waterloo, Ontario, Canada, August 14-18, 1998

[11] J. Gotman, J. Zhang, J. Rosenblatt, and R. Gotman, Evaluation of an automatic seizure detection method for the newborn EEG Electroencephalogr. Clin. Neurophysiol., vol.103,pp.363-369, 1997.

[12] G. Czarnota, M. Kolios, M. Sherar, F. Ottensmeyer, and J. Hunt High-frequency ultrasound imaging of apoptosis in vitro, in situ and in vivo. Faseb Journal. Vol. 13 (7), pp. A1436-A1436. 1999.

[13] A. Liu, J.S.Hahn, G.P.Heldt, and R.W. Coen, Detection of neonatal seizures through computerized EEG analysis Electroencephalogr. Clin. Neurophysiol., vol. 82, pp. $30-37,1992$.

[14] B. Friedlander and B. Porat. The modified Yule-Walker method of ARMA spectral estimation. IEEE Trans. Aerospace Electronic Syst. AES-20(2), pp.158-173, 1984.

[15] W. Kang, J. Shiu, C. Cheng, J. Lai, H. Tsao and T. Kuo. The application of Cepstral Coefficients and Maximum Likelihood Method in EMG Pattern recognition IEEE Trans. on Biomedical Engineering. Vol. 42 (8), pp. 777-785, August 1995. 
[16] G. Czarnota, M. Kolios, M. Sherar, F. Ottensmeyer, and J. Hunt. High-frequency ultrasound imaging of apoptosis in vitro, in situ and in vivo. Faseb Journal. Vol. 13 (7), pp. A1436-A1436. 1999.

[17] B. Boashash Time-frequency signal analysis, in Advances in Spectral Estimation and Array Processing Prentice Hall, NJ, 1991, pp. 418 - 517

[18] L. B. White and B. Boashash Cross Spectral analysis of non-stationary processes. IEEE Trans. Informat. Theory, vol. 36, pp. 830 - 835, July 1990

[19] W. S. Pritchard and D. W. Duke Measuring chaos in the brain: A tutorial review of nonlinear dynamical EEG analysis Intern. J. Neuroscience, vol. 67, pp. 31 - 80, 1992.

[20] M. J. A. M. van Putten and C. J. Stam Application of a neural complexity measure to multi-channel EEG Phys. Letters A, vol. 281/2-3 pp. 131 - 141, 2001.

[21] P. L. Nunez et al. EEG coherency I: Statistics, reference electrode, volume conduction, Laplacians, cortical imaging, and interpretation at multiple scales Electroencephalogr. Clin. Neurophysiol., vol. 103, pp. 499 - 486, 1999.

[22] A. Babloyantz, J. M. Salazar, and C. Nicolis, Evidence of chaotic dynamics of brain activity during the sleep cycle Physics Letters A, vol. 222, pp. 152 - 156, 1985. 